\title{
Hit-and-run for numerical integration
}

Daniel Rudolf

\begin{abstract}
We study the numerical computation of an expectation of a bounded function $f$ with respect to a measure given by a non-normalized density on a convex body $K \subset \mathbb{R}^{d}$. We assume that the density is log-concave, satisfies a variability condition and is not too narrow. In [19, 25, 26] it is required that $K$ is the Euclidean unit ball. We consider general convex bodies or even the whole $\mathbb{R}^{d}$ and show that the integration problem satisfies a refined form of tractability. The main tools are the hit-and-run algorithm and an error bound of a multi run Markov chain Monte Carlo method.
\end{abstract}

\section{Introduction and results}

In many applications, for example in Bayesian inference, see [5, 8], or in statistical physics, see [18, 27], it is desirable to compute an expectation of the form

$$
\int_{K} f(x) \pi_{\rho}(\mathrm{d} x)=\int_{K} f(x) c \rho(x) \mathrm{d} x,
$$

where the probability measure $\pi_{\rho}$ is given by the density $c \rho$ with $c>0$. The normalizing constant of the density

$$
\frac{1}{c}=\int_{K} \rho(x) \mathrm{d} x
$$

is not known and hard to compute. We want to have algorithms that are able to compute the expectation without any precompution of $c$.

Dr. Daniel Rudolf

Friedrich-Schiller-University Jena, Institute of Mathematics, Ernst-Abbe-Platz 2, 07743 Jena, Germany. e-mail: daniel.rudolf@uni-jena.de 
More precisely, let $\rho: \mathbb{R}^{d} \rightarrow \mathbb{R}_{+}$be a possibly non-normalized density function, let $K=\operatorname{supp}(\rho) \subset \mathbb{R}^{d}$ be a convex body and let $f: K \rightarrow \mathbb{R}$ be integrable with respect to $\pi_{\rho}$. For a tuple $(f, \rho)$ we define the desired quantity

$$
A(f, \rho)=\frac{\int_{K} f(x) \rho(x) \mathrm{d} x}{\int_{K} \rho(x) \mathrm{d} x} .
$$

In [19] a simple Monte Carlo method is considered which evaluates the numerator and denominater of $A(f, \rho)$ on a common independent, uniformly distributed sample in $K$. There it must be assumed that one can sample the uniform distribution in $K$. The authors show that this algorithm is not able to use any additional structure, such as log-concavity, of the density function. But they show that such structure can be used by Markov chain Monte Carlo which then outperforms the simple Monte Carlo method.

Markov chain Monte Carlo algorithms for the integration problem of the form (11) are considered in [19, 21, 25, 26]. Basically it is always assumed that $K$ is the Euclidean unit ball rather than a general convex body. We extend the results to the case where $K$ might even be the whole $\mathbb{R}^{d}$ if the density satisfies some further properties. We do not assume that we can sample with respect to $\pi_{\rho}$. The idea is to compute $A(f, \rho)$ by using a Markov chain which approximates $\pi_{\rho}$. We prove that the integration problem (1) satisfies an extended type of tractability. Now let us introduce the error criterion and the new notion of tractability.

Error criterion and algorithms. Let $t: \mathbb{N} \times \mathbb{N} \rightarrow \mathbb{N}$ be a function and let $A_{n, n_{0}}$ be a generic algorithm which uses $t\left(n, n_{0}\right)$ Markov chain steps. Intuitively, the number $n_{0}$ determines the number of steps to approximate $\pi_{\rho}$. The number $n$ determines the number of pieces of information of $f$ used by the algorithm. The error is measured in mean square sense, for a tuple $(f, \rho)$ it is given by

$$
e\left(A_{n, n_{0}}(f, \rho)\right)=\left(\mathrm{E}\left|A_{n, n_{0}}(f, \rho)-A(f, \rho)\right|^{2}\right)^{1 / 2},
$$

where $\mathrm{E}$ denotes the expectation with respect to the joint distribution of the used sequence of random variables determined by the Markov chain.

For example the algorithm might be a single or multi run Markov chain Monte Carlo. More precisely, assume that we have a Markov chain with limit distribution $\pi_{\rho}$ and let $X_{1}, \ldots, X_{n+n_{0}}$ be the first $n+n_{0}$ steps. Then

$$
S_{n, n_{0}}(f, \rho)=\frac{1}{n} \sum_{j=1}^{n} f\left(X_{j+n_{0}}\right)
$$

is an approximation of $A(f, \rho)$ and the function $t\left(n, n_{0}\right)=n+n_{0}$. In contrast to the single run Markov chain Monte Carlo $S_{n, n_{0}}$ one might consider a multi run Markov chain Monte Carlo, say $M_{n, n_{0}}$, given as follows. Assume that we have $n$ independent Markov chains with the same transition kernel, the same initial distribution and limit 
distribution $\pi_{\rho}$. Let $X_{n_{0}}^{1}, \ldots, X_{n_{0}}^{n}$ be the sequence of the $n_{0}$ th steps of the Markov chains, then

$$
M_{n, n_{0}}(f, \rho)=\frac{1}{n} \sum_{j=1}^{n} f\left(X_{n_{0}}^{j}\right)
$$

is an approximation of $A(f, \rho)$. In this setting the function $t\left(n, n_{0}\right)=n \cdot n_{0}$.

Tractability. In [19, 21] a notion of tractability for the integration problem (1) is introduced. It is assumed that $\|f\|_{\infty} \leq 1$ and that the density function satisfies

$$
\frac{\sup _{x \in K} \rho(x)}{\inf _{x \in K} \rho(x)} \leq \gamma,
$$

for some $\gamma \geq 3$. Let $s_{\varepsilon, \gamma}\left(n, n_{0}\right)$ be the minimal number of function values of $(f, \rho)$ to guarantee an $\varepsilon$-approximation with respect to the error above. Then the integration problem is called tractable with respect to $\gamma$ if $s_{\varepsilon, \gamma}\left(n, n_{0}\right)$ depends polylogarithmically on $\gamma$ and depends polynomially on $\varepsilon^{-1}, d$. We extend this notion of tractability. We study a class of tuples $(f, \rho)$ which satisfy $\|f\|_{\infty} \leq 1$ and we assume that for any $\rho$ there exists a set $G \subset K$ such that for $\kappa \geq 3$ holds

$$
\frac{\int_{K} \rho(x) \mathrm{d} x}{\operatorname{vol}_{d}(G) \inf _{x \in G} \rho(x)} \leq \kappa,
$$

where $\operatorname{vol}_{d}(G)$ denotes the $d$-dimensional volume of $G$. Then we call the integration problem tractable with respect to $\kappa$ if the minimal number of function values $t_{\varepsilon, \kappa}\left(n, n_{0}\right)$ of $(f, \rho)$ to guarantee an $\varepsilon$-approximation satisfies for some non-negative numbers $p_{1}, p_{2}$ and $p_{3}$ that

$$
t_{\varepsilon, \kappa}\left(n, n_{0}\right)=\mathscr{O}\left(\varepsilon^{-p_{1}} d^{p_{2}}[\log \kappa]^{p_{3}}\right), \quad \varepsilon>0, d \in \mathbb{N}, \kappa \geq 3 .
$$

Hence we permit only polylogarithmical dependence on the number $\kappa$, since it might be very large (e.g. $10^{30}$ or $10^{40}$ ). The extended notion of tractability allows us to consider $K=\operatorname{supp}(\rho)=\mathbb{R}^{d}$.

The structure of the work and the main results are as follows. We use the hitand-run algorithm to approximate $\pi_{\rho}$. An explicit estimate of the total variation distance of the hit-and-run algorithm, proven by Lovász and Vempala in [15, 16], and an error bound of the mean square error of $M_{n, n_{0}}$ are essential. In Section 2 we provide the basics on Markov chains and prove an error bound of $M_{n, n_{0}}$. In Section3 we define the class of density functions. Roughly we assume that the densities are log-concave, that for any $\rho$ there exists a set $G \subset K$ such that condition (2) holds for $\kappa \geq 3$ and that the densities are not too narrow. Namely, we assume that level sets of $\rho$ of measure larger than $1 / 8$ contain a ball with radius $r$. We distinguish two settings which guarantee that the densities are not too spread out. Either the convex body $K=\operatorname{supp}(\rho)$ is bounded by a ball with radius $R$ around 0 , then we say $\rho \in \mathscr{U}_{r, R, \kappa}$, or the support of $\rho$ is bounded in average sense, 


$$
\int_{K}\left|x-x_{\rho}\right|^{2} \pi_{\rho}(\mathrm{d} x) \leq 4 R^{2}
$$

where $x_{\rho}=\int_{K} x \pi_{\rho}(\mathrm{d} x) \in \mathbb{R}^{d}$ is the centroid. Then we say $\rho \in \mathscr{V}_{r, R, K}$. For precise definitions see Section 3 . In Section 4 we provide the hit-and-run algorithm and state convergence properties of the algorithm for densities from $\mathscr{U}_{r, R, K}$ and $\mathscr{V}_{r, R, K}$. Then we show that the integration problem (1) is tractable with respect to $\kappa$, see Section 5 For $\rho \in \mathscr{U}_{r, R, K}$ we obtain in Theorem 4 that

$$
t_{\varepsilon, \kappa}\left(n, n_{0}\right)=\mathscr{O}\left(d^{2}[\log d]^{2} \varepsilon^{-2}\left[\log \varepsilon^{-1}\right]^{3}[\log \kappa]^{3}\right) .
$$

For $\rho \in \mathscr{V}_{r, R, \kappa}$ we find in Theorem[5 a slightly worse bound of the form

$$
t_{\varepsilon, \kappa}\left(n, n_{0}\right)=\mathscr{O}\left(d^{2}[\log d]^{2} \varepsilon^{-2}\left[\log \varepsilon^{-1}\right]^{5}[\log \kappa]^{5}\right) .
$$

Here the $\mathscr{O}$ notation hides the polynomial dependence on $r$ and $R$.

In [19, 21, 25, 26] it is proven that the problem (1) is tractable with respect to $\gamma$ for $K=B_{d}$, where $B_{d}$ denotes the Euclidean unit ball. Note that for $G=B_{d}$ we have

$$
\frac{\int_{K} \rho(x) \mathrm{d} x}{\operatorname{vol}_{d}(G) \inf _{x \in G} \rho(x)} \leq \frac{\sup _{x \in K} \rho(x)}{\inf _{x \in K} \rho(x)} \leq \gamma .
$$

Furthermore it is assumed that $\rho: B_{d} \rightarrow \mathbb{R}_{+}$is $\log$-concave and $\log \rho$ is Lipschitz. Then the Metropolis algorithm with a ball walk proposal is used to approximate $\pi_{\rho}$. For $\|f\|_{p} \leq 1$ with $p>2$ the algorithm $S_{n, n_{0}}$ is considered for the approximation of $A(f, \rho)$. It is proven that

$$
s_{\varepsilon, \gamma}\left(n, n_{0}\right)=\mathscr{O}\left(d \max \left\{\log ^{2}(\gamma), d\right\}\left(\varepsilon^{-2}+\log \gamma\right)\right) .
$$

In open problem 84 of [21] it is asked whether one can extend this result to other families of convex sets. The complexity bound of (5) is better than the results of (3) and (4) in terms of the dimension, the precision and $\gamma$. On the one hand the assumption that $K=B_{d}$ is very restrictive but on the other hand the estimates of (3) and (4) seem to be pessimistic. However, with our results we contribute to problem 84 in the sense that tractability with respect to $\gamma$ can be shown for arbitrary convex bodies or even the whole $\mathbb{R}^{d}$ if the density functions satisfy certain properties.

\section{Markov chains and an error bound}

Let $\left(X_{n}\right)_{n \in \mathbb{N}}$ be a Markov chain with transition kernel $P(\cdot, \cdot)$ and initial distribution $v$ on a measurable space $(K, \mathscr{B}(K))$, where $K \subset \mathbb{R}^{d}$ and $\mathscr{B}(K)$ is the Borel $\sigma$-algebra. We assume that the transition kernel $P(\cdot, \cdot)$ is reversible with respect to $\pi_{\rho}$. For $p \in[1, \infty]$ we denote by $L_{p}=L_{p}\left(\pi_{\rho}\right)$ the class of functions $f: K \rightarrow \mathbb{R}$ with 


$$
\|f\|_{p}=\left(\int_{K}|f(x)|^{p} \pi_{\rho}(\mathrm{d} x)\right)^{1 / p}<\infty .
$$

Similarly we denote by $\mathscr{M}_{p}$ the class of measures $v$ which are absolutely continuous with respect to $\pi_{\rho}$ and where the density $\frac{d v}{d \pi_{\rho}} \in L_{p}$. The transition kernel induces an operator $P: L_{p} \rightarrow L_{p}$ given by

$$
P f(x)=\int_{K} f(y) P(x, \mathrm{~d} y), \quad x \in K,
$$

and it induces an operator $P: \mathscr{M}_{p} \rightarrow \mathscr{M}_{p}$ given by

$$
\mu P(C)=\int_{K} P(x, C) \mu(\mathrm{d} x), \quad C \in \mathscr{B}(K) .
$$

For $n \in \mathbb{N}$ and a probability measure $v$ note that $\operatorname{Pr}\left(X_{n} \in C\right)=v P^{n}(C)$, where $C \in$ $\mathscr{B}(K)$. We define the total variation distance between $v P^{n}$ and $\pi_{\rho}$ as

$$
\left\|v P^{n}-\pi_{\rho}\right\|_{\mathrm{tv}}=\sup _{C \in \mathscr{B}(K)}\left|v P^{n}(C)-\pi_{\rho}(C)\right| .
$$

Under suitable assumptions on the Markov chain one obtains that $\left\|v P^{n}-\pi_{\rho}\right\|_{\mathrm{tv}} \rightarrow$ 0 as $n \rightarrow \infty$.

Now we consider the multi run Markov chain Monte Carlo method and prove an error bound. This bound is not new, see for example [4].

Theorem 1. Assume that we have $n_{0}$ independent Markov chains with transition kernel $P(\cdot, \cdot)$ and initial distribution $v \in \mathscr{M}_{1}$. Let $\pi_{\rho}$ be a stationary distribution of $P(\cdot, \cdot)$. Let $X_{n_{0}}^{1}, \ldots, X_{n_{0}}^{n}$ be the sequence of the $n_{0}$ th steps of the Markov chains and let

$$
M_{n, n_{0}}(f, \rho)=\frac{1}{n} \sum_{j=1}^{n} f\left(X_{n_{0}}^{j}\right)
$$

Then

$$
e\left(M_{n, n_{0}}(f, \rho)\right)^{2} \leq \frac{1}{n}\|f\|_{\infty}^{2}+2\|f\|_{\infty}^{2}\left\|v P^{n}-\pi_{\rho}\right\|_{t v} .
$$

Proof. With an abuse of notation let us denote

$$
A(f)=\int_{K} f(x) \pi_{\rho}(\mathrm{d} x) \quad \text { and } \quad v P^{n_{0}}(f)=\int_{K} f(x) v P^{n_{0}}(\mathrm{~d} x) .
$$

We decompose the error into variance and bias. Then

$$
\begin{aligned}
e\left(M_{n, n_{0}}(f, \rho)\right)^{2} & =\frac{1}{n} \int_{K}\left|f(x)-v P^{n_{0}}(f)\right|^{2} v P^{n_{0}}(\mathrm{~d} x)+\left|v P^{n_{0}}(f)-A(f)\right|^{2} \\
& =\frac{1}{n}\left(v P^{n_{0}}\left(f^{2}\right)-v P^{n_{0}}(f)^{2}\right)+\left|v P^{n_{0}}(f)-A(f)\right|^{2} \\
& \leq \frac{1}{n}\|f\|_{\infty}^{2}+\int_{K} f(x)^{2}\left|v P^{n_{0}}(\mathrm{~d} x)-\pi_{\rho}(\mathrm{d} x)\right|
\end{aligned}
$$




$$
\leq \frac{1}{n}\|f\|_{\infty}^{2}+2\|f\|_{\infty}^{2}\left\|v P^{n_{0}}-\pi_{\rho}\right\|_{\mathrm{tv}} .
$$

The last inequality follows by a well known characterization of the total variation distance, see for example [24, Proposition 3].

Very often there exists a number $\beta \in[0,1)$ and a number $C_{v}<\infty$ such that

$$
\left\|v P^{n}-\pi_{\rho}\right\|_{\mathrm{tv}} \leq C_{v} \beta^{n} .
$$

For example, if $\beta=\|P-A\|_{L_{2} \rightarrow L_{2}}<1$ and $C_{v}=\frac{1}{2}\left\|v-\pi_{\rho}\right\|_{2}$, see [23] for more details. Let us define the $L_{2}$-spectral gap as

$$
\operatorname{gap}(P)=1-\|P-A\|_{L_{2} \rightarrow L_{2}} .
$$

This is a significant quantity, see for instance [2, 26, 27, 28, 29]. In [26] it is shown that

$$
e\left(S_{n, n_{0}}(f, \rho)\right)^{2} \leq \frac{4\|f\|_{4}}{n \operatorname{gap}(P)} \quad \text { for } \quad n_{0} \geq \frac{\log \left(64\left\|\frac{d v}{d \pi_{\rho}}-1\right\|_{2}\right)}{\operatorname{gap}(P)} .
$$

There are several Markov chains where it is possible to provide, for certain classes of density functions, a lower bound of $\operatorname{gap}(P)$ which grows polynomially with respect to the dimension, see for example [16, 19]. Then, the error bound of the single run Markov chain Monte Carlo method might imply that the integration problem (11) is tractable with respect to some $\kappa$.

Note that there are also other possible approximation schemes and other bounds of the error of $S_{n, n_{0}}$ which depend on different assumptions to the Markov chain (e.g. Ricci curvature condition, drift condition, small set), see for instance [9, 11, 12, 13]. For example one might consider a multi run Markov chain Monte Carlo method where function values of a trajectory of each Markov chain after a sufficiently large $n_{0}$ are used. But all known error bounds of such methods include quantities such as the $L_{2}$-spectral gap or the conductance.

It is not an easy task to prove that a Markov chain satisfies the different assumptions stated above and it is also not an easy task to prove a lower bound of the $L_{2}$-spectral gap. It might be easier to estimate the total variation distance of $v P^{n_{0}}$ and $\pi_{\rho}$ directly. Then one can use Theorem 1 to show that the integration problem (1) is tractable with respect to some $\kappa$.

\section{Densities with additional structure}

Let us assume that the densities have some additional structure. For $0<r \leq R$ and $\kappa \geq 3$ a density function $\rho: K \rightarrow \mathbb{R}_{+}$is in $\mathscr{U}_{r, R, \kappa}$ if the following properties are satisfied: 
(a) $\rho$ is $\log$-concave, i.e. for all $x, y \in K$ and $\lambda \in[0,1]$ one has

$$
\rho(\lambda x+(1-\lambda) y) \geq \rho(x)^{\lambda} \rho(y)^{1-\lambda} .
$$

(b) $\rho$ is strictly positive, i.e. $K=\operatorname{supp}(\rho)$ and we assume that $K \subset R B_{d}$, where $R B_{d}$ is the Euclidean ball with radius $R$ around 0 .

(c) There exists a set $G \subset K$ such that

$$
\frac{\int_{K} \rho(x) \mathrm{d} x}{\operatorname{vol}_{d}(G) \inf _{x \in G} \rho(x)} \leq \kappa,
$$

and we can sample the uniform distribution on $G$.

(d) For $s>0$ let $K(s)=\{x \in K \mid \rho(x) \geq t\}$ be the level set of $\rho$ and let $B(z, r)$ be the Euclidean ball with radius $r$ around $z$. Then

$$
\pi_{\rho}(K(s)) \geq \frac{1}{8} \quad \Longrightarrow \quad \exists z \in K \quad B(z, r) \subset K(s) .
$$

The log-concavity of $\rho$ implies that the maximal value is attained on a convex set, that the function is continuous and that one has an isoperimetric inequality, see [16]. Assumption (b) gives that $K$ is bounded.

By (C) we can sample the uniform distribution on $G$. We can choose it as initial distribution for a Markov chain, where the number $\kappa$ provides an estimate of the influence of this initial distribution.

The condition on the level set $K(s)$ guarantees that the peak is not too narrow. Roughly speaking, if the $\pi_{\rho}$ measure of a level set is not too small, then the Lebesgue measure is also not too small. Note that $K$ is bounded from below, since condition (d) implies that $B(z, r) \subset K$.

Now we enlarge the class of densities. Let us define the following property:

(b') $\rho$ is strictly positive, i.e. $K=\operatorname{supp}(\rho)$ and $x_{\rho}=\int_{K} x \pi_{\rho}(\mathrm{d} x) \in \mathbb{R}^{d}$ is the centroid of $\pi_{\rho}$. Then

$$
\int_{K}\left|x-x_{\rho}\right|^{2} \pi_{\rho}(\mathrm{d} x) \leq 4 R^{2}
$$

We have $\rho \in \mathscr{V}_{r, R, \kappa}$ if the density $\rho$ satisfies (a), (b) ( (C) and (d). We substituted the boundedness condition (b) by (b). Note that (b) implies (b). Hence $\mathscr{U}_{r, R, \kappa} \subset \mathscr{V}_{r, R, K}$. Condition $\left({ }^{b}\right)$ provides a boundedness criterion in average sense. Namely, it implies that

$$
\int_{K} \int_{K}|x-y|^{2} \pi_{\rho}(\mathrm{d} x) \pi_{\rho}(\mathrm{d} y) \leq 8 R^{2}
$$

Example of a Gaussian function in $\mathscr{V}_{r, R, \kappa}$. Let $\Sigma$ be a symmetric and positive definite $d \times d$ matrix. We consider the non-normalized density

$$
\varphi(x)=\exp \left(-\frac{1}{2} x^{T} \Sigma^{-1} x\right), \quad x \in \mathbb{R}^{d} .
$$


The target distribution $\pi_{\varphi}$ is a normal distribution with mean $x_{\varphi}=0 \in \mathbb{R}^{d}$ and covariance matrix $\Sigma$. There exists an orthogonal matrix $V=\left(v_{1}, \ldots, v_{d}\right)$, where $v_{1}, \ldots, v_{d}$ are the eigenvectors of $\Sigma$. Then

$$
V^{-1} \Sigma V=\Lambda,
$$

where $\Lambda=\operatorname{diag}\left(\lambda_{1}, \ldots, \lambda_{d}\right)$ and $\lambda_{1}, \ldots, \lambda_{d}$ with $\lambda_{i}>0$ for $i \in\{1, \ldots, d\}$ are the corresponding eigenvalues of $\Sigma$. Recall that the trace and the determinant of $\Sigma$ are

$$
\operatorname{tr}(\Sigma)=\sum_{i=1}^{d} \lambda_{i} \quad \text { and } \quad \operatorname{det}(\Sigma)=\prod_{i=1}^{d} \lambda_{i}
$$

We show that if $r, R$ and $\kappa$ are appropriately chosen, then $\varphi \in \mathscr{V}_{r, R, \kappa}$.

To (a): The density $\varphi$ is obviously log-concave.

To (b): Since $x_{\varphi}=0$ we obtain

$$
\int_{K}\left|x-x_{\varphi}\right|^{2} \pi_{\varphi}(\mathrm{d} x)=\frac{1}{(2 \pi)^{d / 2} \sqrt{\operatorname{det}(\Sigma)}} \int_{\mathbb{R}^{d}}|x|^{2} \varphi(x) \mathrm{d} x=\operatorname{tr}(\Sigma) .
$$

Hence we set $R=\frac{1}{2} \sqrt{\operatorname{tr}(\Sigma)}$.

To (ㄷ): Let $\lambda_{\min }=\min _{i=1, \ldots, d} \lambda_{i}$ and let $v_{\min }$ be the corresponding eigenvector. Note that $x^{T} \Sigma^{-1} x \leq \lambda_{\min }^{-1}|x|^{2}$ and that equality holds for $x=v_{\min }$. With $G=B_{d}$ we obtain

$$
\frac{\int_{\mathbb{R}^{d}} \varphi(x) \mathrm{d} x}{\operatorname{vol}_{d}\left(B_{d}\right) \inf _{x \in B_{d}} \varphi(x)}=\exp \left(\frac{1}{2} \lambda_{\text {min }}^{-1}\right) \Gamma(d / 2+1) 2^{d / 2} \sqrt{\operatorname{det}(\Sigma)},
$$

where $\Gamma(d)=\int_{0}^{\infty} t^{d-1} \exp (-t) \mathrm{d} t$ is the gamma function. Hence we set

$$
\kappa=\exp \left(\frac{1}{2} \lambda_{\min }^{-1}\right) \Gamma(d / 2+1) 2^{d / 2} \sqrt{\operatorname{det}(\Sigma)} .
$$

To (d): The level sets of $\varphi$ are ellipsoids

$$
K(s)=\left\{x \in \mathbb{R}^{d} \mid x^{T} \Sigma^{-1} x \leq 2 \log \left(s^{-1}\right)\right\}, \quad s \in[0,1] .
$$

In general one has

$$
\pi_{\varphi}(K(s))=\frac{\int_{0}^{\infty} \operatorname{vol}_{d}(K(s) \cap K(t)) \mathrm{d} t}{\int_{0}^{\infty} \operatorname{vol}_{d}(K(t)) \mathrm{d} t}=\frac{s \operatorname{vol}_{d}(K(s))+\int_{s}^{\infty} \operatorname{vol}_{d}(K(t)) \mathrm{d} t}{\int_{0}^{\infty} \operatorname{vol}_{d}(K(t)) \mathrm{d} t} .
$$

By the well known formula of the volume of an ellipsoid we obtain

$$
\operatorname{vol}_{d}(K(t))=2^{d / 2} \log ^{d / 2}\left(t^{-1}\right) \sqrt{\operatorname{det}(\Sigma)} \operatorname{vol}_{d}\left(B_{d}\right), \quad t \in[0,1]
$$

and 


$$
\pi_{\varphi}(K(s))=\frac{s \log ^{d / 2}\left(s^{-1}\right)+\int_{s}^{1} \log ^{d / 2}\left(t^{-1}\right) \mathrm{d} t}{\int_{0}^{1} \log ^{d / 2}\left(t^{-1}\right) \mathrm{d} t}, \quad s \in[0,1] .
$$

Hence

$$
\pi_{\varphi}(K(s))=\frac{\gamma\left(\log s^{-1}, d / 2\right)}{\Gamma(d / 2)}, \quad s \in[0,1],
$$

where $\gamma(r, d)=\int_{0}^{r} t^{d-1} \exp (-t) \mathrm{d} t$ is the lower incomplete gamma function. Let us define a function $r^{*}: \mathbb{N} \rightarrow \mathbb{R}$ by

$$
r^{*}(d)=\inf \left\{r \in[0, \infty): \gamma(r, d / 2) \geq \frac{1}{8} \Gamma(d / 2)\right\} .
$$

If we substitute $1 / 8$ by $1 / 2$ in the definition of $r^{*}(d)$ we have the median of the gamma distribution with parameter $d / 2$ and 1 . It is known that the median is in $\Theta(d)$, see [1]. Figure 1] suggests that $r^{*}(d)$ behaves also linearly in $d$.

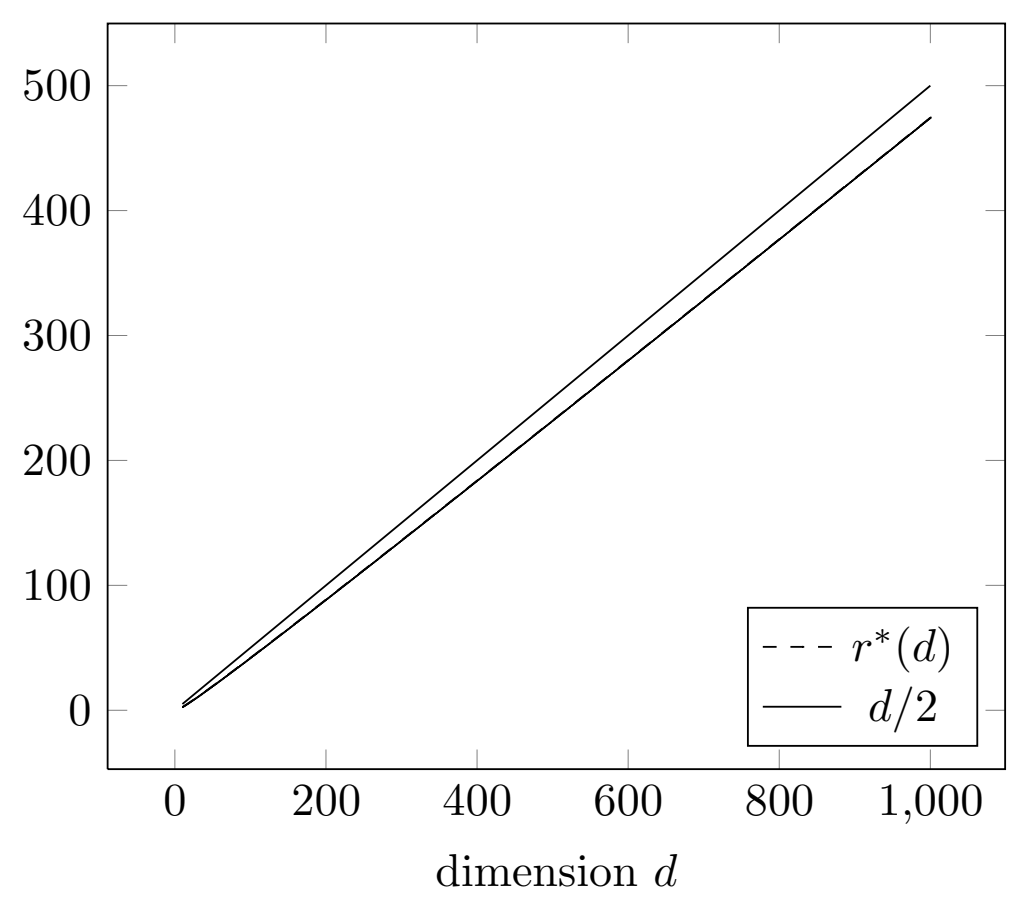

Fig. 1 Plot of an approximation of $r^{*}(d)$ with a Newton method and an appropriately chosen initial value.

Let $\log \left(s^{*}(d)^{-1}\right)=r^{*}(d)$, such that $s^{*}(d)=\exp \left(-r^{*}(d)\right)$. Then

$$
\pi_{\varphi}\left(K\left(s^{*}(d)\right)\right)=\frac{1}{8} \quad \text { and } \quad B\left(0,\left(\lambda_{\min } r^{*}(d)\right)^{1 / 2}\right) \subset K\left(s^{*}(d)\right) .
$$


Hence we set $r=\left(\lambda_{\min } r^{*}(d)\right)^{1 / 2}$.

Let us summarize. For $r=\left(\lambda_{\min } r^{*}(d)\right)^{1 / 2}, R=\frac{1}{2} \sqrt{\operatorname{tr}(\Sigma)}$ and

$$
\kappa=\exp \left(\frac{1}{2} \lambda_{\min }^{-1}\right) \Gamma(d / 2+1) 2^{d / 2} \sqrt{\operatorname{det}(\Sigma)}
$$

we obtain that $\varphi \in \mathscr{V}_{r, R, \kappa}$. Note that $\kappa$ depends exponentially on the dimension $d$. However, if one has tractability with respect to $\kappa$, then the error depends polynomially on the dimension.

\section{Hit-and-run algorithm}

For $\rho: K \rightarrow \mathbb{R}_{+}$the hit-and-run algorithm is as follows. Let $v$ be a probability measure on $(K, \mathscr{B}(K))$ and let $x_{1} \in K$ be chosen by $v$. For $k \in \mathbb{N}$ suppose that the states $x_{1}, \ldots, x_{k}$ are already computed. Then

1. choose a direction $u$ uniformly distributed on $\partial B_{d}$;

2. set $x_{k+1}=x_{k}+\alpha u$, where $\alpha \in I_{k}=\left\{\alpha \in \mathbb{R} \mid x_{k}+\alpha u \in K\right\}$ is chosen with respect to the distribution determined by the density

$$
\ell_{k}(s)=\frac{\rho\left(x_{k}+s u\right)}{\int_{I_{k}} \rho\left(x_{k}+t u\right) \mathrm{d} t}, \quad s \in I_{k} .
$$

The second step might cause implementation issues. However, if we have a logconcave density $\rho$ then $\ell_{k}$ is also log-concave. In this setting one can use different acceptance/rejection methods. For more details see for example [6, Section 2.4.2] or [17]. In the following we assume that we can sample the distribution determined by $\ell_{k}$.

Other algorithms for the approximation of $\pi_{\rho}$ would be a Metropolis algorithm with suitable proposal [19] or a combination of a hit-and-run algorithm with uniform stationary distribution and a Ratio-of-uniforms method [10]. Also hybrid samplers are promising methods, especially when $\rho$ decreases exponentially in the tails [7].

Now let us state the transition kernel, say $H_{\rho}$, of the hit-and-run algorithm

$$
H_{\rho}(x, C)=\frac{2}{\operatorname{vol}_{d-1}\left(\partial B^{d}\right)} \int_{C} \frac{\rho(y) \mathrm{d} y}{\ell_{\rho}(x, y)|x-y|^{d-1}}, \quad x \in K, C \in \mathscr{B}(K),
$$

where

$$
\ell_{\rho}(x, y)=\int_{-\infty}^{\infty} \rho(\lambda x+(1-\lambda) y) \mathbf{1}_{K}(\lambda x+(1-\lambda) y) \mathrm{d} \lambda .
$$

The transition kernel $H_{\rho}$ is reversible with respect to $\pi_{\rho}$, let us refer to [3] for further details. 
In the following we state several results from Lovaśz and Vempala. This part is based on [15]. We start with a special case of [15, Theorem 1.1] and sketch the proof of this theorem.

Theorem 2. Let $\varepsilon \in(0,1 / 2)$ and $\rho \in \mathscr{U}_{r, R, K}$. Let $v$ be an initial distribution with the following property. There exists a set $S_{\varepsilon} \subset K$ and a number $D \geq 1$ such that

$$
\frac{d v}{d \pi_{\rho}}(x) \leq D, \quad x \in K \backslash S_{\varepsilon},
$$

where $v\left(S_{\varepsilon}\right) \leq \varepsilon$. Then for

$$
n_{0}>10^{27}\left(d r^{-1} R\right)^{2} \log ^{2}\left(8 D d r^{-1} R \varepsilon^{-1}\right) \log \left(4 D \varepsilon^{-1}\right)
$$

the total variation distance between $v H_{\rho}^{n_{0}}$ and $\pi_{\rho}$ is less than $2 \varepsilon$.

Proof (Sketch).

1. Let us assume that $S_{\varepsilon}=\emptyset$ :

Then it follows $\left\|\frac{d v}{d \pi_{\rho}}\right\|_{\infty} \leq D$, so that $v \in \mathscr{M}_{\infty}$. We use [14, Corollary 1.6] with $s=\frac{\varepsilon}{2 D}$ and obtain

$$
\left\|v H_{\rho}^{n}-\pi_{\rho}\right\|_{\mathrm{tv}} \leq \varepsilon / 2+D \exp \left(-\frac{1}{2} n \Phi_{\frac{\varepsilon}{2 D}}^{2}\right),
$$

where $\Phi_{\frac{\varepsilon}{2 D}}$ is the $\frac{\varepsilon}{2 D}$-conductance of $H_{\rho}$. By Theorem 3.7 of [15] and the scaling invariance of the hit-and-run algorithm we find a lower bound of $\Phi_{\frac{\varepsilon}{2 D}}$. It is given by

$$
\Phi_{\frac{\varepsilon}{2 D}} \geq \frac{10^{-13}}{2 d r^{-1} R \log \left(4 d r^{-1} R D \varepsilon^{-1}\right)} .
$$

This leads to

$$
\left\|v H_{\rho}^{n}-\pi_{\rho}\right\|_{\mathrm{tv}} \leq \varepsilon / 2+D \exp \left(\frac{-10^{-26} n}{8\left(d r^{-1} R\right)^{2} \log ^{2}\left(4 d r^{-1} R D \varepsilon^{-1}\right)}\right) .
$$

2. Now let us assume that $S_{\varepsilon} \neq \emptyset$ :

Let $\tilde{\varepsilon}:=v\left(S_{\mathcal{\varepsilon}}\right)$, so that $0<\tilde{\varepsilon} \leq \varepsilon \leq 1 / 2$ and for $C \in \mathscr{B}(K)$ let

$$
\mu_{1}(C)=\frac{v\left(C \cap S_{\varepsilon}^{c}\right)}{v\left(S_{\varepsilon}^{c}\right)} \quad \text { and } \quad \mu_{2}(C)=\frac{v\left(C \cap S_{\varepsilon}\right)}{v\left(S_{\varepsilon}\right)} .
$$

Then

$$
v=(1-\tilde{\varepsilon}) \mu_{1}+\tilde{\varepsilon} \mu_{2}
$$

and $\left\|\frac{d \mu_{1}}{d \pi_{\rho}}\right\|_{\infty} \leq 2 D$. Furthermore for any $C \in \mathscr{B}(K)$ we find

$$
\left|v H_{\rho}^{n}(C)-\pi_{\rho}(C)\right| \leq(1-\tilde{\varepsilon})\left|\mu_{1} H_{\rho}^{n}(C)-\pi_{\rho}(C)\right|+\tilde{\varepsilon} .
$$


By using (7) we get

$$
\left\|\mu_{1} H_{\rho}^{n}-\pi_{\rho}\right\|_{\mathrm{tV}} \leq \varepsilon / 2+2 D \exp \left(\frac{-10^{-26} n}{8\left(d r^{-1} R\right)^{2} \log ^{2}\left(8 d r^{-1} R D \varepsilon^{-1}\right)}\right),
$$

and altogether

$$
\left\|v H_{\rho}^{n}-\pi_{\rho}\right\|_{\mathrm{tv}} \leq 3 \varepsilon / 2+2 D \exp \left(\frac{-10^{-26} n}{8\left(d r^{-1} R\right)^{2} \log ^{2}\left(8 d r^{-1} R D \varepsilon^{-1}\right)}\right) .
$$

Choosing $n$ so that the right hand side of the previous equation is less than or equal to $2 \varepsilon$ completes the proof.

The next Corollary provides an explicit upper bound of the total variation distance.

Corollary 1. Under the assumptions of Theorem 2 with

$$
\beta=\exp \left(\frac{-10^{-9}}{\left(d r^{-1} R\right)^{2 / 3}}\right) \quad \text { and } \quad C=12 d r^{-1} R D
$$

one obtains

$$
\left\|v H_{\rho}^{n_{0}}-\pi_{\rho}\right\|_{t v} \leq C \beta \sqrt[3]{n_{0}}, \quad n \in \mathbb{N}
$$

Proof. Set $\varepsilon=8 d r^{-1} R D \exp \left(\frac{-10^{-9} n^{1 / 3}}{\left(d r^{-1} R\right)^{2 / 3}}\right)$ and use (8) to complete the proof.

Note that the result of Theorem 2 is better than the result of Corollary 1 However, Corollary 1 provides an explicit estimate of the total variation distance. One can see that there is an almost exponential decay, namely the total variation distance goes to zero at least as $\beta \sqrt[3]{n_{0}}$ goes to zero for increasing $n_{0}$.

In the previous results we assumed that $\rho \in \mathscr{U}_{r, R, \kappa}$. It is essentially used that (b) holds. Now let us assume that $\rho \in \mathscr{V}_{r, R, \kappa}$. The next statement is proven in [15. Theorem 1.1].

Theorem 3. Let $\varepsilon \in(0,1 / 2), \rho \in \mathscr{V}_{r, R, \kappa}$. Let $v$ be an initial distribution with the following property. There exists a set $S_{\varepsilon} \subset K$ and a number $D \geq 1$ such that

$$
\frac{d v}{d \pi_{\rho}}(x) \leq D, \quad x \in K \backslash S_{\varepsilon}
$$

where $v\left(S_{\varepsilon}\right) \leq \varepsilon$. Then for

$$
n_{0} \geq 4 \cdot 10^{30}\left(d r^{-1} R\right)^{2} \log ^{2}\left(2 D d r^{-1} R \varepsilon^{-1}\right) \log ^{3}\left(D \varepsilon^{-1}\right)
$$

the total variation distance between $v H_{\rho}^{n_{0}}$ and $\pi_{\rho}$ is less than $2 \varepsilon$.

Note that Theorem 2 and Theorem 3 can be applied if the initial distribution is bounded, i.e. we can set $D=\left\|\frac{d v}{d \pi_{\rho}}\right\|_{\infty}$ and $S_{\varepsilon}=\emptyset$. Furthermore if $v \in \mathscr{M}_{2}$, i.e. 
$\left\|\frac{d v}{d \pi_{\rho}}\right\|_{2}$ is bounded, then we can also apply Theorem 2 and Theorem 3 with $D=$ $\left\|\frac{d v}{d \pi_{\rho}}\right\|_{2}^{2} \varepsilon^{-1}$ and

$$
S_{\varepsilon}=\left\{x \in K \mid \frac{d v}{d \pi_{\rho}}(x)>\left\|\frac{d v}{d \pi_{\rho}}\right\|_{2}^{2} \varepsilon^{-1}\right\} .
$$

\section{Main results}

Now we are able to state and to prove the main results. To avoid any pathologies we assume that $r^{-1} R d \geq 3$.

Theorem 4. Let $\varepsilon \in(0,1 / 2)$ and

$$
\mathscr{F}_{r, R, K}=\left\{(f, \rho) \mid \rho \in \mathscr{U}_{r, R, K},\|f\|_{\infty} \leq 1\right\} .
$$

For $(f, \rho) \in \mathscr{F}_{r, R, \kappa}$ let $v$ be the uniform distribution on $G \subset \mathbb{R}^{d}$ from (ㄷ). Let $X_{n_{0}}^{1}, \ldots, X_{n_{0}}^{n}$ be a sequence of the $n_{0}$ th steps of $n$ independent hit-and-run Markov chains with stationary distribution $\pi_{\rho}$ and initial distribution $v$. Recall that

$$
M_{n, n_{0}}(f, \rho)=\frac{1}{n} \sum_{j=1}^{n} f\left(X_{n_{0}}^{j}\right) .
$$

Then for $n \geq \varepsilon^{-2}$ and

$$
n_{0} \geq 10^{27}\left(d r^{-1} R\right)^{2} \log ^{2}\left(8 d r^{-1} R \kappa \varepsilon^{-2}\right) \log \left(4 \kappa \varepsilon^{-2}\right)
$$

we obtain

$$
\sup _{(f, \rho) \in \mathscr{F}_{r, R, K}} e\left(M_{n, n_{0}}(f, \rho)\right) \leq 3 \varepsilon
$$

Hence

$$
t_{\varepsilon, \kappa}\left(n, n_{0}\right)=\mathscr{O}\left(d^{2}\left(r^{-1} R\right)^{2} \log ^{2}\left(d r^{-1} R\right) \varepsilon^{-2}\left[\log \varepsilon^{-1}\right]^{3}[\log \kappa]^{3}\right) .
$$

Proof. For $C \in \mathscr{B}(K)$ we have

$$
v(C)=\int_{C} \frac{\mathbf{1}_{G}(y) \int_{K} \rho(x) \mathrm{d} x}{\operatorname{vol}_{d}(G) \rho(y)} \pi_{\rho}(\mathrm{d} y) .
$$

It implies that $\frac{d v}{d \pi_{\rho}}(x) \leq \kappa$ for all $x \in K$. Then the assertion follows by Theorem 1 and Theorem 2

Now let us consider densities which belong to $\mathscr{V}_{r, R, \kappa}$.

Theorem 5. Let $\varepsilon \in(0,1 / 2)$ and 


$$
\mathscr{G}_{r, R, \kappa}=\left\{(f, \rho) \mid \rho \in \mathscr{V}_{r, R, \kappa},\|f\|_{\infty} \leq 1\right\} .
$$

Let $M_{n, n_{0}}$ be given as in Theorem 4 Then for $n \geq \varepsilon^{-2}$ and

$$
n_{0} \geq 4 \cdot 10^{30}\left(d r^{-1} R\right)^{2} \log ^{2}\left(2 d r^{-1} R \kappa \varepsilon^{-2}\right) \log ^{3}\left(\kappa \varepsilon^{-2}\right)
$$

we obtain

$$
\sup _{(f, \rho) \in \mathscr{G}_{r, R, K}} e\left(M_{n, n_{0}}(f, \rho)\right) \leq 3 \varepsilon
$$

Hence

$$
t_{\varepsilon, \kappa}\left(n, n_{0}\right)=\mathscr{O}\left(d^{2}\left(r^{-1} R\right)^{2} \log ^{2}\left(d r^{-1} R\right) \varepsilon^{-2}\left[\log \varepsilon^{-1}\right]^{5}[\log \kappa]^{5}\right) .
$$

Proof. The assertion follows by the same steps as the proof of Theorem 4 Note that we use Theorem 3 instead of Theorem 2 .

Note that in both theorems there is no hidden dependence on further parameters in the $\mathscr{O}$ notation. However, the explicit constant might be very large, of the magnitude of $10^{30}$. The theorems imply that the problem of integration (1) is tractable with respect to $\kappa$ on the classes $\mathscr{F}_{r, R, \kappa}$ and $\mathscr{G}_{r, R, \kappa}$.

Example of a Gaussian function revisited. In the Gaussian example of Section 3 we obtained

$$
\begin{aligned}
R / r & =\left(2 r^{*}(d)^{1 / 2}\right)^{-1} \cdot \sqrt{\operatorname{tr}(\Sigma) / \lambda_{\min }}, \\
\kappa & =\exp \left(\frac{1}{2} \lambda_{\min }^{-1}\right) \Gamma(d / 2+1) 2^{d / 2} \sqrt{\operatorname{det}(\Sigma)} .
\end{aligned}
$$

If we assume that $r^{*}(d)$ increases linearly in $d$ (Figure 1), that $\sqrt{\operatorname{tr}(\Sigma) / \lambda_{\min }}$ and $\log \left(\exp \left(\frac{1}{2} \lambda_{\min }^{-1}\right) \sqrt{\operatorname{det}(\Sigma)}\right)$ grows polynomially in the dimension, then $t_{\varepsilon, \kappa}\left(n, n_{0}\right)$ grows also polynomially in the dimension. This implies that the integration problem with respect to the Gaussian function is polynomially tractable in the sense of Novak and Woźniakowski [20, 21, 22].

Acknowledgements The author gratefully acknowledges the comments of the referees and wants to express his thanks to the local organizers of the Tenth International Conference on Monte Carlo and Quasi-Monte Carlo Methods in Scientific Computing for their hospitality. The research was supported by the DFG Priority Program 1324 and the DFG Research Training Group 1523.

\section{References}

1. Adell, J., Jodrá, P.: Sharp estimates for the median of the $\Gamma(n+1,1)$ distribution. Statist. Probab. Lett. 71(2), 185-191 (2005) 
2. Aldous, D.: On the Markov chain simulation method for uniform combinatorial distributions and simulated annealing. Probability in the Engineering and Informational Sciences 1(1), 33-46 (1987)

3. Bélisle, C., Romeijn, E., Smith, R.: Hit-and-run algorithms for generating multivariate distributions. Math. Oper. Res. 18(2), 255-266 (1993)

4. Belloni, A., Chernozhukov, V.: On the computational complexity of MCMC-based estimators in large samples. Ann. Statist. 37(4), 2011-2055 (2009)

5. Brooks, S., Gelman, A., Jones, G., Meng, X.: Handbook of Markov chain Monte Carlo. Chapman \& Hall (2011)

6. Casella, G., Robert, C.: Monte Carlo statistical methods, second edn. Springer Texts in Statistics. Springer-Verlag, New York (2004)

7. Fort, G., Moulines, E., Roberts, G., Rosenthal, J.: On the geometric ergodicity of hybrid samplers. J. Appl. Probab. 40(1), 123-146 (2003)

8. Gilks, W., Richardson, S., Spiegelhalter, D.: Markov chain Monte Carlo in practice. Chapman \& Hall (1996)

9. Joulin, A., Ollivier, Y.: Curvature, concentration and error estimates for Markov chain Monte Carlo. Ann. Probab. 38(6), 2418-2442 (2010)

10. Karawatzki, R., Leydold, J., Potzelberger, K.: Automatic Markov chain Monte Carlo procedures for sampling from multivariate distributions. Tech. Rep. 27, Department of Statistics and Mathematics, WU Wien (2005)

11. Łatuszyński, K., Miasojedow, B., Niemiro, W.: Nonasymptotic bounds on the estimation error of MCMC algorithms. ArXiv e-prints (2011)

12. Łatuszyński, K., Miasojedow, B., Niemiro, W.: Nonasymptotic bounds on the mean square error for MCMC estimates via renewal techniques. ArXiv e-prints (2011)

13. Łatuszyński, K., Niemiro, W.: Rigorous confidence bounds for MCMC under a geometric drift condition. J. Complexity 27(1), 23-38 (2011)

14. Lovász, L., Simonovits, M.: Random walks in a convex body and an improved volume algorithm. Random Structures and Algorithms 4(4), 359-412 (1993)

15. Lovász, L., Vempala, S.: Fast algorithms for logconcave functions: sampling, rounding, integration and optimization. In: Proceedings of the 47th Annual IEEE Symposium on Foundations of Computer Science, FOCS '06, pp. 57-68. IEEE Computer Society, Washington, DC, USA (2006)

16. Lovász, L., Vempala, S.: Hit-and-run from a corner. SIAM J. Comput. 35(4), 985-1005 (2006)

17. Lovász, L., Vempala, S.: The geometry of logconcave functions and sampling algorithms. Random Structures Algorithms 30(3), 307-358 (2007)

18. Martinelli, F.: Relaxation times of Markov chains in statistical mechanics and combinatorial structures. In: Probability on discrete structures, Encyclopaedia Math. Sci., vol. 110, pp. 175262. Springer, Berlin (2004)

19. Mathé, P., Novak, E.: Simple Monte Carlo and the Metropolis algorithm. J. Complexity 23(46), 673-696 (2007)

20. Novak, E., Woźniakowski, H.: Tractability of multivariate problems. Vol. 1: Linear information, EMS Tracts in Mathematics, vol. 6. European Mathematical Society (EMS), Zürich (2008)

21. Novak, E., Woźniakowski, H.: Tractability of multivariate problems. Vol. 2: Standard information for functionals, EMS Tracts in Mathematics, vol. 12. European Mathematical Society (EMS), Zürich (2010)

22. Novak, E., Woźniakowski, H.: Tractability of multivariate problems. Vol. 3: Standard information for Operators, EMS Tracts in Mathematics, vol. 12. European Mathematical Society (EMS), Zürich (2012)

23. Roberts, G., Rosenthal, J.: Geometric ergodicity and hybrid Markov chains. Electron. Comm. Probab. 2, no. 2, 13-25 (1997)

24. Roberts, G., Rosenthal, J.: General state space Markov chains and MCMC algorithms. Probability Surveys 1, 20-71 (2004)

25. Rudolf, D.: Explicit error bounds for lazy reversible Markov chain Monte Carlo. J. Complexity 25(1), 11-24 (2009) 
26. Rudolf, D.: Explicit error bounds for Markov chain Monte Carlo. Dissertationes Math. 485, 93 pp. (2012)

27. Sokal, A.: Monte Carlo methods in statistical mechanics: foundations and new algorithms. In: Functional integration (Cargèse, 1996), NATO Adv. Sci. Inst. Ser. B Phys., vol. 361, pp. 131-192. Plenum, New York (1997)

28. Ullrich, M.: Comparison of Swendsen-Wang and heat-bath dynamics. ArXiv e-prints (2011)

29. Ullrich, M.: Swendsen-wang is faster than single-bond dynamics. ArXiv e-prints (2012) 